\title{
Application of linear polarized light for the discrimination of frozen and liquid droplets in ice nucleation experiments
}

\author{
T. Clauss ${ }^{1}$, A. Kiselev ${ }^{1,2}$, S. Hartmann ${ }^{1}$, S. Augustin ${ }^{1}$, S. Pfeifer ${ }^{1}$, D. Niedermeier ${ }^{1}$, H. Wex ${ }^{1}$, and F. Stratmann ${ }^{1}$ \\ ${ }^{1}$ Leibniz Institute for Tropospheric Research, Permoserstr. 15, 04318 Leipzig, Germany \\ ${ }^{2}$ Institute for Meteorology and Climate Research, Karlsruhe Institute of Technology, Postfach 3640, \\ 76021 Karlsruhe, Germany
}

Correspondence to: T. Clauss (tina.clauss@tropos.de)

Received: 22 July 2012 - Published in Atmos. Meas. Tech. Discuss.: 20 August 2012

Revised: 6 March 2013 - Accepted: 22 March 2013 - Published: 19 April 2013

\begin{abstract}
We report on the development and test results of the new optical particle counter TOPS-Ice (Thermostabilized Optical Particle Spectrometer for the detection of Ice). The instrument uses measurements of the crosspolarized scattered light by single particles into the nearforward direction $\left(42.5^{\circ} \pm 12.7^{\circ}\right)$ to distinguish between spherical and non-spherical particles. This approach allows the differentiation between liquid water droplets (spherical) and ice particles (non-spherical) having similar volumeequivalent sizes and therefore can be used to determine the fraction of frozen droplets in a typical immersion freezing experiment. We show that the numerical simulation of the light scattered on non-spherical particles (spheroids in random orientation) considering the actual scattering geometry used in the instrument supports the validity of the approach, even though the cross-polarized component of the light scattered by spherical droplets does not vanish in this scattering angle. For the separation of the ice particle mode from the liquid droplet mode, we use the width of the pulse detected in the depolarization channel instead of the pulse height. Exploiting the intrinsic relationship between pulse height and pulse width for Gaussian pulses allows us to calculate the fraction of frozen droplets even if the liquid droplet mode dominates the particle ensemble. We present test results obtained with TOPS-Ice in the immersion freezing experiments at the laminar diffusion chamber LACIS (Leipzig Aerosol Cloud Interaction Simulator) and demonstrate the excellent agreement with the data obtained in similar experiments with a different optical instrument. Finally, the advantages of using the cross-polarized light measurements for the differentiation of liquid and frozen droplets in the realistic immersion freezing experiments are discussed.
\end{abstract}

\section{Introduction}

Ice particles in clouds strongly affect the cloud dynamics and radiative properties and therefore the Earth's climate (Cantrell and Heymsfield, 2005). Whereas in hightropospheric clouds ice forms mostly by direct deposition from the vapor phase onto solid aerosol particles, ice particles in mixed-phase tropospheric clouds tend to form by freezing of liquid droplets condensed on the preexisting solid particles called ice nuclei (IN). In this context, an important freezing mechanism under atmospheric conditions is immersion freezing (Ansmann et al., 2008; Prenni et al., 2009; Wiacek et al., 2010; de Boer et al., 2011), where supercooled droplets with immersed insoluble particles freeze. These particles lower the free-energy barrier associated with the formation of an ice germ of critical size in the supercooled liquid water and therefore dramatically enhance the rate of supercooled droplet freezing, leading to higher onset temperature of ice formation (which is approximately $-38^{\circ} \mathrm{C}$ for homogeneous freezing of supercooled water droplets; Pruppacher and Klett, 1997).

However, the physical and chemical properties of aerosol particles that are responsible for their ability to serve as heterogeneous ice nuclei are not entirely understood. Among other qualities, the affinity of the crystalline structure of mineral dust to the crystalline structure of ice, the presence of $\mathrm{OH}$ radicals on the surface of organic IN, and a special protein on the surface of biological particles are considered. To elucidate these unknowns, laboratory studies involving wellcharacterized reference IN are required.

Among others, the ongoing research in this field is done in the ice nucleation chambers of the continuous flow diffusion 
type (e.g., LACIS, Stratmann et al., 2004; Hartmann et al., 2011; CFDC, Rogers, 1988; FINCH, Bundke et al., 2008; ZINC, Stetzer et al., 2008) and in the expansion cloud chambers (e.g., AIDA, Wagner et al., 2009), where liquid droplets with immersed IN are cooled down to a temperature where some fraction of them freezes.

The majority of modern ice nucleation instruments measure the ice fraction $f_{\text {ice }}$, i.e., the number of ice particles divided by the total number of particles (ice particles and droplets), as a function of temperature, size, and microphysical properties of IN. The value of $f_{\text {ice }}$ is a measure of the freezing ability of supercooled droplets. The ability to measure this property correctly depends strongly on the ability of a particle-counting instrument to distinguish between liquid droplets and ice particles. This is typically done by optical means, exploiting the fact that at water saturation ice crystals grow faster than liquid droplets, thus making a simple size-threshold-based segregation of detected particles possible (Rogers, 1988; Wagner et al., 2009).

In recent immersion freezing measurements with the Leipzig Aerosol Cloud Interaction Simulator (LACIS) described in Niedermeier et al. $(2010,2011)$, the commercial white light optical particle spectrometer (WELAS 1000, Palas, Karlsruhe, Germany) was used for the determination of the ice fraction. In these experiments, LACIS was operated in a mode where the non-frozen supercooled droplets evaporated, leaving ice and dry particles at the outlet of the flow tube. If the dry aerosol particles are much smaller than the ice particles, the latter can be clearly distinguished from the dry particles, and so $f_{\text {ice }}$ can be calculated. In certain situations, however, the dry aerosol particles have a broad size distribution overlapping with the size distribution of ice particles. The only way to resolve such a mixture is to allow the coexistence of ice particles and water droplets and to apply a different method to separate both populations of particles. This is a challenging task when both water droplets and ice particles have approximately the same optical size, making the size-based separation impossible.

In this case, the geometrical difference between the highly spherical liquid droplets and the non-spherical ice particles has to be exploited. The most common approach is based on the fact that light scattered by spherical particles in the nearbackward direction preserves its polarization state, while non-spherical particles change the state of polarization depending on the particle shape and orientation. This technique has been successfully used in remote sensing applications, for example, in lidar polarimetric measurements of ice and mixed-phase clouds, e.g., in Sassen (1991), Ansmann et al. (2009), and Seifert et al. (2010).

Studying the scattering of polarized light on non-spherical particles (spheroids), Mishchenko and Sassen (1998) and Zakharova and Mishchenko (2000) have shown that even a small increase of the aspect ratio and, therefore, a small deviation of the particle from the spherical shape can lead to a significantly large increase of the depolarization ratio. At the same time, no systematic dependence of the depolarization ratio on the degree of non-sphericity has been found. Taking into account the variability of ice crystal habits in atmospheric clouds (Bailey and Hallett, 2009), the applicability of the polarimetric approach has to be demonstrated in practice. Until now, only few in-situ instruments exist using the measurement of the polarization state of the scattered light for the discrimination of spherical and non-spherical particles (dust) (Glen and Brooks, 2013) or the discrimination of ice and water in laboratory and field experiments (Bundke et al., 2008; Krämer et al., 2009; Nicolet et al., 2010; Schnaiter et al., 2012).

From different prototype stages of the Thermo-stabilized Optical Particle Spectrometer for the detection of Ice (TOPSIce) development, we decided not to use the scattering in the backward direction, first, to avoid the different fields of view of the three detectors used in TOPS-Ice (two for the size discrimination in the forward direction, and one for the waterice discrimination) and, second, to increase the scattering intensity. Motivated by the need to build an optical instrument capable of counting ice particles in a system containing both liquid and frozen droplets of similar sizes, and trying to avoid the complications connected with the measuring of light scattered into the near-backward direction, we have investigated, both experimentally and theoretically by numerical calculations, the possibility of using the polarimetric measurements of light scattered in the near-forward direction.

In the following, we show the development of TOPS-Ice coupled with LACIS. The differentiation algorithm, which uses the pulse width of the cross-polarized scattering pulse of a particle streaming out of LACIS, will be explained and verified with theoretical calculations of the scattering intensity for different particle types.

\section{Experimental setup}

In the following section, the experimental setup for the immersion freezing experiments is described. The particle conditioning, i.e., the generation of droplets with a single immersed solid particle and the freezing of these droplets, takes place in the cloud simulator LACIS, which is described in Sect. 2.1. The optical particle spectrometer, TOPS-Ice, which was developed to detect the particles at the outlet of LACIS, is described in Sect. 2.2.

\subsection{LACIS}

The heterogeneous ice nucleation experiments are performed in the laminar flow diffusion chamber LACIS, which is explicitly described in Stratmann et al. (2004) and Hartmann et al. (2011). LACIS is a $7 \mathrm{~m}$ long vertical flow tube with an internal diameter of $15 \mathrm{~mm}$. Mobility-selected aerosol particles are fed into LACIS through the inlet located on top of the flow tube. The humidified aerosol flow streams 
into the tube with a flow velocity of $0.4 \mathrm{~m} \mathrm{~s}^{-1}$ forming an aerosol stream of $2 \mathrm{~mm}$ in diameter in the center of the tube, which is surrounded by particle-free humidified sheath air. LACIS consists of seven $1 \mathrm{~m}$ long separate sections. By adjusting the wall temperatures of the sections, a precise temperature and saturation profile can be established along the tube axis. As a result, all particles moving along the axis of the LACIS flow tube experience the same humidity and temperature conditions. In the experiments described in this section, LACIS was operated in immersion freezing mode (Niedermeier et al., 2010); i.e., the seed particles were first activated to supercooled droplets, and then the droplets were cooled down to the temperature where some of them freeze. For a certain amount of these supercooled droplets, the immersed particles act as IN, leading to heterogeneous ice nucleation and hence freezing of the droplet. The number of frozen droplets divided by the number of frozen and liquid droplets yields the ice fraction $f_{\text {ice }}$, which can be considered as the probability of heterogeneous freezing as a function of temperature, particle type, particle size, and time.

\subsection{TOPS-Ice}

To determine the fraction of ice particles in the presence of liquid droplets in LACIS, we have developed TOPS-Ice (Thermo-Stabilized Optical Particle Spectrometer for the detection of Ice). The layout of the instrument is shown in Fig. 1. TOPS-Ice uses a diode-pumped solid state continuous wave laser (LasNova 50 green GLK 3220 T01, LASOS Lasertechnik GmbH, Jena, Germany) with a wavelength of $532 \mathrm{~nm}$ and an output power of $20 \mathrm{~mW}$ as a light source. The laser beam has a Gaussian profile with a width of $0.7 \mathrm{~mm}$ (FWHM) and emits linearly polarized light, vertically polarized $(200: 1)$ (normal to the drawing plane in Fig. 1). The beam is enlarged to a width of $2 \mathrm{~mm}$ and flattened by a cylindrical lens to intensify the illumination within the sensitive volume; thus the resulting beam is $2 \mathrm{~mm}$ in width and about $24 \mu \mathrm{m}$ in height (see the discussion in Sect. 4.1). The purity in terms of linear polarization of the laser beam is ensured by means of a Glan-Thompson calcite polarizer (extinction ratio: $100000: 1$ ). The laser beam is focused on the particles streaming out of LACIS. The particles move through the illuminated volume and scatter the light into all directions; the transmitted laser beam is intercepted by a beam trap. The light scattered by individual particles is coupled into three optical fibers (with a light acceptance cone of $25.4^{\circ}$ ) into the solid angle of $0.15 \mathrm{sr}$ located around the scattering angle $\vartheta=42.5^{\circ}$. The optical fibers are used to deliver the scattered light to three different photomultiplier tubes (PMT, Hamamatsu H6780-04). PMT A detects light at a scattering angle of $\left(\vartheta=42.5^{\circ}, \varphi=180^{\circ}\right)$, and PMT B and PMT C detect the scattered light at an angle of $\left(\vartheta=42.5^{\circ}, \varphi=0\right)$, where $\vartheta$ is the scattering angle and $\varphi$ is the azimuth angle. The core diameter of the fibers is $400 \mu \mathrm{m}$ and $200 \mu \mathrm{m}$ for PMT A and PMT B/C, respectively. A non-polarizing beam

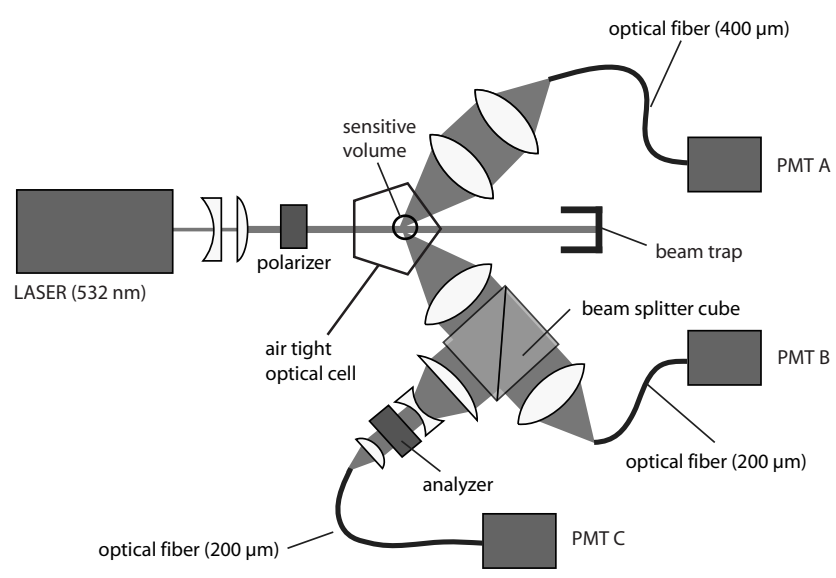

Fig. 1. Optical layout of TOPS-Ice (top view).

splitter cube $(1: 1)$ within the optical pathway of PMT B allows the detection of light by a third detector, PMT C, within the same angular range. The three detectors therefore possess the same detector geometry. In front of PMT C, another Glan-Thompson polarizer transmits only the horizontally polarized component (parallel to the drawing plane) of the scattered light. Around the outlet of LACIS, an air-tight optical cell is built, so that the laser light and the scattered light are coupled out and in, respectively, through glass windows. The cooling jacket of LACIS protrudes inside the optical cell, ensuring that the temperature of the gas flow stays constant down to the sensitive volume (and explaining the "thermostabilized" in the name of the instrument). The larger core diameter of the optical fiber for PMT A allows for a larger field of view for PMT A as compared to PMT B/C, so that the sensitive volume, shaped by the intersection of the illumination beam and the field of view of PMT B/C, is embedded into the sensitive volume of PMT A. This is done to minimize edge zone errors. A particle is only counted as being "valid" if all detectors receive a signal at the same time to ensure that the particle is situated completely within the sensitive volume caused by the intersection of the illumination beam and PMT A. To record and analyze the scattering pulses by the three detectors and to provide an almost real-time validation of the measured signal, we use a fast data acquisition unit together with a LabVIEW-based program package, as described in Kiselev et al. (2005).

The validated pulse amplitudes received from PMT A can then be used to retrieve the size distribution of the droplets. This is achieved by means of the instrument response function that is first calculated theoretically for the TOPS-Ice scattering geometry and then calibrated with monodisperse polystyrene latex microspheres (PSL, Duke Scientific Corp., Palo Alto, CA, USA) within a size range of $250 \mathrm{~nm}$ and $1600 \mathrm{~nm}$ as described in Kiselev et al. (2005). An example of the response curve for TOPS-Ice is shown in Fig. 4. Although the curve is ambiguous and oscillates strongly, the 
uncertainty in the size determination is less than $0.5 \mu \mathrm{m}$ for spherical particles. The differentiation of water droplets and ice particles was tested for sizes larger than $1000 \mathrm{~nm}$.

The scattered light detected with PMT C is used to distinguish between frozen and unfrozen droplets. To discriminate between the spherical and the non-spherical particles, we exploit the property of light scattered by spherical droplets to preserve the original polarization state within the scattering plane. This is generally not true for the non-spherical particles. The measurement of the cross-polarized component can therefore be exploited for distinguishing between spherical and non-spherical particles. The theoretical background is given in Sect. 3, while the applied differentiation algorithm based on the measurement of the pulse width of the scattered pulse is explained in Sect. 4.

\section{Theory}

Different approaches for the theoretical investigation of the scattering of ice particles with different habits have been developed; the most widely used being geometric ray-tracing methods (e.g., Takano and Jayaweera, 1985; Macke et al., 1996), the finite difference time domain method (FDTD) (e.g., Yang and Liou, 1996; Baran et al., 2001), the Tmatrix approach (e.g., Mishchenko and Hovenier, 1995; Mishchenko and Sassen, 1998; Rother, 2009) and the discrete dipole approximation (DDA) (e.g., Draine and Flatau, 1994). Meanwhile, a wide database of computational programs exists for the theoretical investigation of the scattering behavior for diverse particle shapes (Wriedt and Hellmers, 2008). An application of the T-matrix code from Mishchenko and Sassen (1998) for orientated cylindrical ice particles was published recently by Nicolet et al. (2012).

The purpose of the following calculations is to show the scattered intensity for spheres and for spheroids, as an example of non-spherical particles, for the exact detector geometry of TOPS-Ice. For the calculations, we used orientationaveraged spheroids. This is possible as the conditions in LACIS do not change over time and allow, therefore, an interpretation of many single particles in random orientation. Thus, the average amplitude of the cross-polarized scattering component can be calculated for an ensemble of orientationaveraged non-spherical particles and can be considered as the expected value.

For the following considerations, the components $P_{i, j}(\vartheta)$ of the Mueller matrix (van de Hulst, 1982; Bohren and Huffmann, 1983) with a resolution of $\Delta \vartheta=1^{\circ}$ were calculated: for randomly oriented spheroids, the T-matrix method from the work package mieschka (Rother, 2009) was used; for spherical particles, as a special case of the spheroids, Lorenz-Mie theory also from the work package mieschka was used. To be able to compare the results for particles with different shape, the volume-equivalent size parameter $x_{\mathrm{ve}}$ is chosen as the characteristic size of the particles.
All data were calculated with a refractive index of $n=1.33$, which corresponds to the refractive index of water at $\lambda=532 \mathrm{~nm}$. We neglect the difference of the refractive indexes of water and ice $(n=1.31)$ since it is irrelevant for the main purpose of our examination.

We use linearly, vertically polarized light, so that the Stokes vector for the incident light has the following form: $S_{\mathrm{i}}=\left(I_{\mathrm{i}}, Q_{\mathrm{i}}, U_{\mathrm{i}}, V_{\mathrm{i}}\right)^{T}=(1,-1,0,0)^{T}$. To calculate the scattered light at an arbitrary observation point on the detector, which is rotated into $42.5^{\circ}$ scattering angle, the effective Mueller matrix $\mathbf{F}(\vartheta, \varphi)$ has to be examined as described in Yang et al. (2003). The parameter $\vartheta$ describes the scattering angle and $\varphi$ is the azimuth angle. The evaluation of $\mathbf{F}(\vartheta, \varphi)$ is done for discrete points in steps of $\Delta \vartheta=1^{\circ}$ and $\Delta \varphi=1^{\circ}$. The resulting Stokes vector for the scattered light $\boldsymbol{S}_{\mathrm{s}}$ as a function of $\vartheta$ and $\varphi$ can then be calculated by the following equation:

$\boldsymbol{S}_{\mathrm{S}}(\vartheta, \varphi)=\left(\begin{array}{c}I_{\mathrm{S}}(\vartheta, \varphi) \\ Q_{\mathrm{s}}(\vartheta, \varphi) \\ U_{\mathrm{s}}(\vartheta, \varphi) \\ V_{\mathrm{S}}(\vartheta, \varphi)\end{array}\right)=\mathbf{F}(\vartheta, \varphi) \boldsymbol{S}_{\mathrm{i}}$

The total scattering intensity as measured by PMT A and PMT B equals to $I_{\mathrm{S}}(\vartheta, \varphi)$. The horizontal component of the scattered intensity as measured by PMT C, which has a polarizer in front, is given by

$I_{\mathrm{X}}(\vartheta, \varphi)=\frac{1}{2}\left(I_{\mathrm{S}}(\vartheta, \varphi)+Q_{\mathrm{S}}(\vartheta, \varphi)\right)$.

To derive the total measured intensity in the angular range of the detector PMT $\mathrm{C} I_{\mathrm{C}}^{\mathrm{d}}$ at $\vartheta=42.5^{\circ} \pm 12.7^{\circ}$, the calculated intensity $I_{\mathrm{X}}(\vartheta, \varphi)$ is integrated over the detector area. A similar approach can be taken to derive the intensity measured by the other detectors $I_{\mathrm{A}, \mathrm{B}}^{\mathrm{d}}$ by integrating $I_{\mathrm{S}}(\vartheta, \varphi)$ over the detector area.

The spherical detector area is defined by a solid angle with half-opening angle of $\beta=12.7^{\circ}$. The center of the detector area is located at $\vartheta_{\mathrm{d}}=42.5^{\circ}$ and $\varphi=0$. To formulate a condition for a point $(\vartheta, \varphi)$ lying within the detector area $A_{\mathrm{d}}$, the coordinate system is rotated so that the point $\left(\vartheta^{\prime}=0, \varphi^{\prime}=0\right)$ in the new coordinate system describes the center of the detector area, and the condition $\vartheta^{\prime} \leq \beta$ describes a point within the detector area. Consequently, the condition for a point $(\vartheta, \varphi)$ in the original coordinate system lying within the solid angle of the detector yields

$\arccos \left(-\sin \vartheta_{\mathrm{d}} \sin \vartheta \cos \varphi+\cos \vartheta_{\mathrm{d}} \cos \vartheta\right) \leq \beta$

As we use discrete values for $\vartheta$ and $\varphi\left(\Delta \vartheta=1^{\circ}, \Delta \varphi=1^{\circ}\right)$, the integral over the detector area has to be replaced with the summation over discretized values $I_{x}$ for every $(\vartheta, \varphi)$ lying within the field of view of the detector (condition from Eq. 3), leading to the intensity $I_{\mathrm{C}}^{\mathrm{d}}$ measured by PMT C: 
$I_{\mathrm{C}}^{\mathrm{d}}=\Delta \vartheta \Delta \varphi \sum_{(\vartheta, \varphi) \subset A_{\mathrm{d}}} I_{\mathrm{x}}(\vartheta, \varphi) \sin \vartheta$

To compare the actual signal detected by PMT $\mathrm{C}$ for spheres and spheroid particles with different aspect ratios $\epsilon$, the intensity $I_{\mathrm{C}}^{\mathrm{d}}$ from Eqs. (3) and (4) is calculated, shown in Fig. 2 for different volume-equivalent size parameters $x_{\mathrm{ve}}$. The considered spheroids have two axes of the same length $a$ and one axis of length $b$. The aspect ratio $\epsilon$ is defined as the ratio $b / a$, so that aspect ratios of $\epsilon<1$ represent oblate spheroids and aspect ratios of $\epsilon>1$ represent prolate spheroids. The axis on top allows for conversion of the volume-equivalent size parameter $x_{\mathrm{ve}}$ to the volumeequivalent particle diameter $d_{\mathrm{ve}}$ for the used wavelength of $532 \mathrm{~nm}$. The axis on the right shows what the calculated relative intensity on the detector approximately means in terms of the voltage signal measured by PMT $\mathrm{C}$. The conversion to the detector voltage signal is performed by a comparison of PMT A and PMT C with signals from water droplets, and PMT A was calibrated with spherical PSL particles with known diameter and refractive index. The figure reveals two main features: first, the cross-polarized component of light scattered by spherical particles is nonzero, and, therefore, the presence of a signal in channel $\mathrm{C}$ cannot be used as a single criteria for detecting the non-spherical particle; and second, the calculated detector response is significantly higher for the randomly oriented spheroids with an aspect ratio $\epsilon \neq 1$ than for a spherical particles $(\epsilon=1)$ of the same volumeequivalent size. Besides, the figure shows a general increase of the signal for larger particles. In brief, assuming comparable sizes and no preferred orientation, a signal-strength-based differentiation between spherical and non-spherical particles is possible. Both assumptions (size similarity of the particle population and the absence of preferential orientation) used for this simulation are realistic for LACIS: the conditions inside the flow tube are well defined; thus, every particle experiences the same saturation and temperature conditions, resulting in similar droplet sizes at the outlet of LACIS, and, assuming the ice particles have only a short time to grow and freeze at approximately the same time, similar ice particle sizes can be assumed; furthermore, for a flow velocity of $0.7 \mathrm{~m} \mathrm{~s}^{-1}$ no preferred orientation can be expected.

The difference between spherical and non-spherical particles is further demonstrated in Fig. 3. Here, the intensity $I_{\mathrm{C}}^{\mathrm{d}}$ on PMT C normalized by $I_{\mathrm{C}}^{\mathrm{d}}$ of the sphere is plotted over the volume-equivalent size parameter $x_{\mathrm{ve}}$ for different aspect ratios $\epsilon$. The signal of the spheroid particles is on average about a factor of 5 higher than for the spherical particles. Therefore, a clear differentiation between the spherical and non-spherical particles in random orientation is possible.

We have to admit, however, that calculating the orientation-averaged scattering signal does not provide ultimate proof that no particle in any fixed orientation would produce a signal comparable to that of a spherical particle

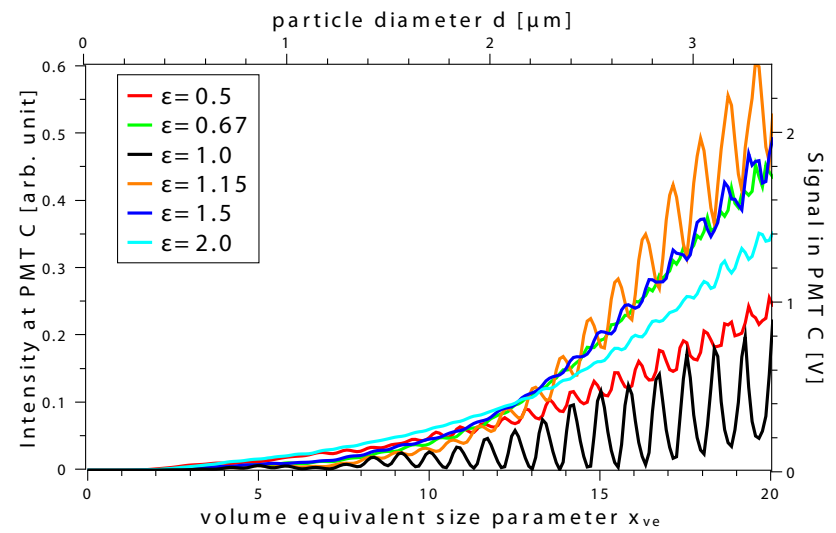

Fig. 2. Intensity integrated over the detector area (PMT C) $I_{\mathrm{C}}^{\mathrm{d}}$ versus volume-equivalent size parameter $x_{\mathrm{ve}}$ for randomly oriented spheroids with different aspect ratios $\epsilon$. Here and in the following figures, the axis on top shows particle diameter corresponding to $x_{\mathrm{ve}}$ (bottom axis) and the wavelength of the TOPS-Ice laser $(\lambda=532 \mathrm{~nm})$. The axis at the right shows the measured signal voltage in PMT C.

having the same $d_{\mathrm{ve}}$. Calculations of the response functions of spheroid particles in all fixed orientations were not performed and would not provide ultimate applicability proof either, considering that the actual ice particles never exhibit rotational symmetry and that the depolarization factor is nonlinearly dependent on the degree of deviation from an ideal sphere (Mishchenko and Sassen, 1998). Note also that a distinction of particle shape (in terms of $\epsilon$ ) is not possible with our ice droplet differentiation method.

In Fig. 4, the relative intensity integrated over the detector area that has no polarizer (PMT A/B) within the optical pathway is shown. In contrast to Fig. 2, the detector response hardly differs for different particle shapes. However, the close resemblance of the response functions for small $\left(x_{\mathrm{ve}}<20\right)$ spherical and non-spherical particles suggests that the response function averaged over $\epsilon$ might be used for the determination of the particle size without introducing a too strongly shape-dependent error.

\section{Ice fraction determination with TOPS-Ice}

Even if TOPS-Ice detects the signal of a single particle, either a droplet or an ice particle, for the data evaluation, the measured values of signal height and width from many single particles are combined in histograms. During the first measurements of $f_{\text {ice }}$ conducted with TOPS-Ice at LACIS, we have discovered that it is beneficial to use the signal width distribution and not the signal height distribution of pulses recorded by PMT C for the droplet and ice particle size range investigated. 


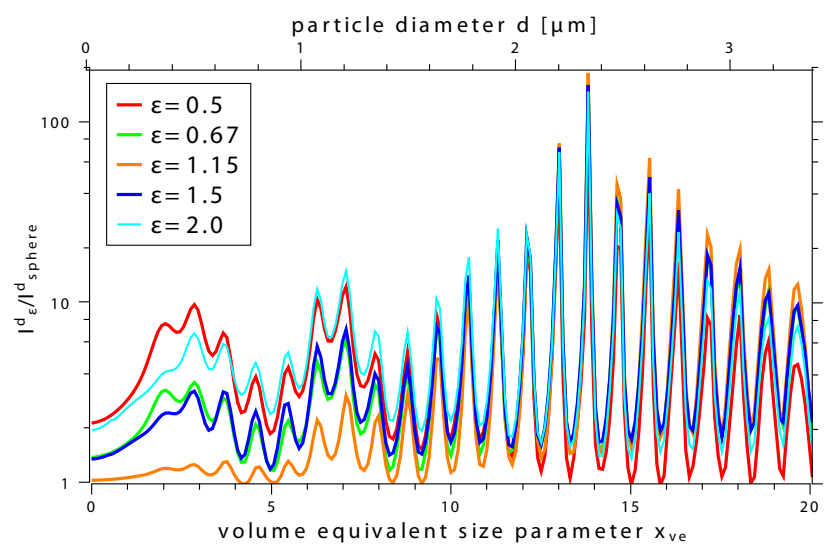

Fig. 3. Intensity on detector area (PMT C) $I_{\mathrm{C}}^{\mathrm{d}}$ from randomly oriented spheroids normalized by $I_{\mathrm{C}}^{\mathrm{d}}$ from sphere volume-equivalent size parameter $x_{\mathrm{ve}}$ for different aspect ratios $\epsilon$.

In addition, it can not be excluded that the size of the ice particles differs from the size of the droplets. Even if the time from the nucleation event until the detection within TOPS-Ice is quite short $(<1.6 \mathrm{~s}$ as the absolute maximum, most ice particles have much less time to grow), the size of the ice particles can be at maximum about double the size of the droplets, depending on the experiment. Additionally, the ice particles have a much broader optical size distribution, measured with PMT A, than the droplets. This is due to the different orientations and depends on the time of the individual nucleation event within LACIS. Depending on the time of nucleation event, the size of the droplets at the nucleation event and the time the ice particles have to grow differs. Therefore, the size of the ice particles also differs in different experiments. However, we can be sure that the ice particles are not smaller than the droplets, and this is always the case within every LACIS freezing experiment with a monodisperse droplet population.

In the following sections, we will discuss the reason for the use of the pulse width distribution and describe the realization of this approach for the retrieval of ice fractions. We also compare the values of $f_{\text {ice }}$ measured with two different instruments, TOPS-Ice and WELAS, for immersion freezing on Arizona Test Dust (ATD).

\subsection{Using the pulse width of the scattering signal for the discrimination of droplets and ice particles}

The analogue voltage signals from the three PMTs are digitized by a 14-bit high-speed waveform digitizer (USB3000, R-Technology Ltd) with a sampling rate of $1 \mathrm{MHz}$ per detection channel and are recorded by the measurement computer. The digital signal is then smoothed by applying a digital filter with a Gaussian kernel to reduce the RMS noise level from $0.06 \mathrm{~V}$ to about $0.01 \mathrm{~V}$. The validation conditions (see Kiselev et al., 2005) are then applied to the recorded

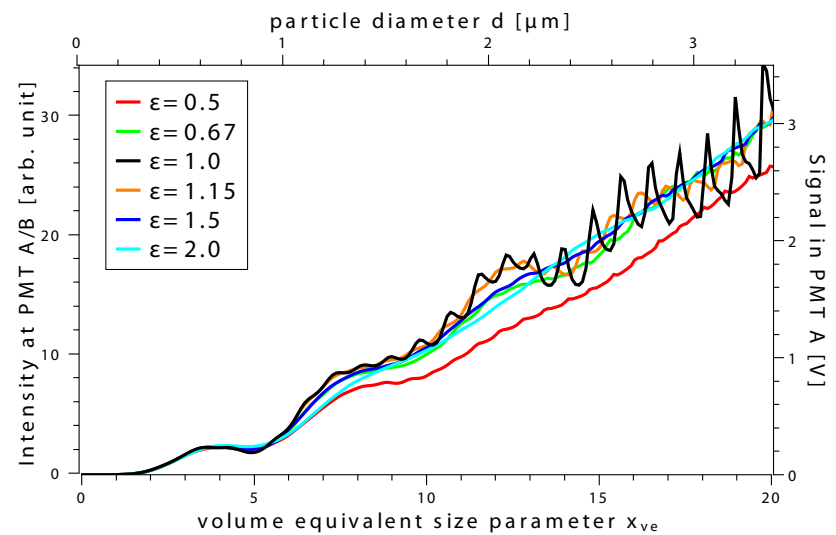

Fig. 4. Intensity integrated over the detector area without a polarizer within the optical pathway (PMT A/B) $I_{\mathrm{A} / \mathrm{B}}^{\mathrm{d}}$ versus volumeequivalent size parameter $x_{\mathrm{ve}}$ for orientation-averaged spheroids with different aspect ratios $\epsilon$.

sequence of pulses. The raw pulse waveforms, i.e., the recorded voltage signal from the detector versus time, generated by a scattering particle moving through the illuminated measurement volume, have an approximately Gaussian shape, reflecting the profile of the laser beam in the vertical direction.

If the smoothed signal exceeds a preset trigger threshold $L$ (usually we use a trigger threshold of $0.03 \mathrm{~V}$ ) for the duration of some minimum time interval for all three detectors, the waveform is considered as a pulse and its parameters (entry time stamp, height, width, maximum signal position) are stored if the validation conditions are fulfilled. The shape of a pulse waveform depends on the spatial distribution of the illumination intensity within the sensitive volume, the velocity and size of the particle, and the delay time of the electronics. For every valid signal pulse, the signal pulse height $H$ and the signal pulse width $W$ from all three detectors are stored. From these data, a pulse height distribution (PHD) and a pulse width distribution (PWD) for an ensemble of particles is recorded. The PHD of PMT A (henceforth PHD ${ }^{\mathrm{A}}$ ) is used to derive a size distribution of the particle ensemble. The PWD of PMT C $\left(\right.$ PWD $\left.^{C}\right)$ is used for the water-ice discrimination; this will be explained below.

To characterize the spatial distribution of the laser intensity in the sensitive volume of TOPS-Ice, we have placed a pinhole into the measurement volume (Fig. 5). The diffraction on the pinhole simulates the scattering of a particle. By displacing the pinhole along the $\mathrm{x}_{-}, \mathrm{y}-$ and $\mathrm{z}$-axis, the illumination intensity within the measurement volume was determined by measuring the response on the different detectors as a function of the pinhole position. Following the method suggested by Schmidt et al. (2004), we recorded the threedimensional response signal distributions across the measurement volume for different pinhole diameters (from $50 \mu \mathrm{m}$ to $200 \mu \mathrm{m}$ ) and extrapolated these intensity distributions 


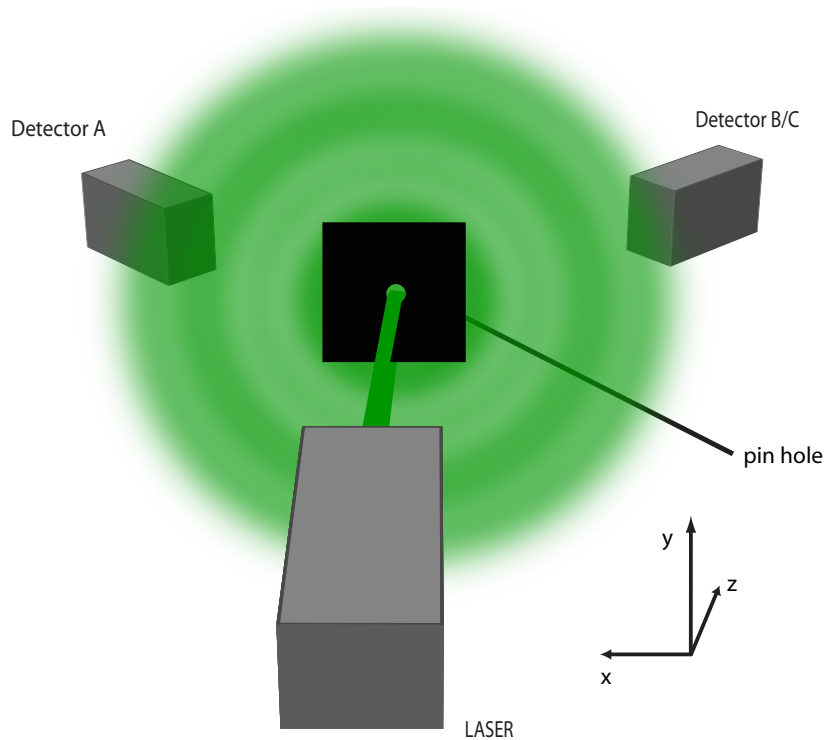

Fig. 5. The experimental setup for the measuring of the sensitive volume. The diffraction on the pinhole simulates the scattering on a particle. The pinhole can be displaced precisely along the $\mathrm{x}-, \mathrm{y}-$ or z-axis.

towards the zero pinhole diameter. Summarizing, the signal profile across the sensitive volume in the y-direction was found to have a Gaussian shape with a constant standard deviation. Additional, the illumination profile was analyzed with a beam profiler and showed a $2 \sigma$-width of $24 \mu \mathrm{m}$.

The usage of the signal pulse width $W$ as a measurement parameter instead of the signal pulse height $H$ turned out to have some advantages in our special measurement regime. Consider a signal pulse that has a Gaussian shape and hence can be described with the following formula:

$P(t)=H \cdot \exp \left(-\frac{\left(t-t_{0}\right)^{2}}{2 \sigma_{\mathrm{p}}^{2}}\right)$,

where $H$ is the signal pulse height, $t_{0}$ is the position of signal maximum on the timescale, and $\sigma_{\mathrm{p}}$ is the standard deviation in units of the timescale ( $\mu$ s). In Fig. 6, an ideal pulse waveform is shown. The values $t_{1}$ and $t_{2}$ are the solutions of the equation $P(t)-L=0$, where $L$ is the trigger threshold, and are given by the following equation:

$t_{1,2}= \pm \sqrt{2 \sigma_{\mathrm{p}}^{2} \log \frac{H}{L}}+t_{0}$.

The pulse width $W$ is defined as $W=t_{2}-t_{1}$ and can be expressed as a function of pulse height $H$ :

$W_{L, \sigma}(H)=2 \sqrt{2 \sigma_{\mathrm{p}}^{2} \log \frac{H}{L}}$,

which is equivalent to

$H_{L, \sigma}(W)=L \exp \frac{1}{2 \sigma^{2}}\left(\frac{W}{2}\right)^{2}$.

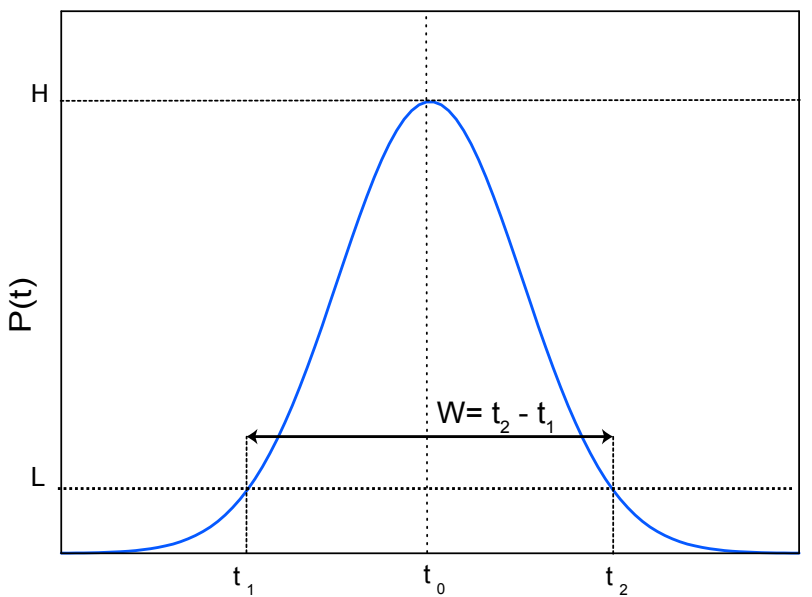

Fig. 6. Waveform of an ideal signal pulse. Parameter described in text.

Substituting the typical values $\sigma_{\mathrm{p}}=28 \mu$ s and $L=0.03 \mathrm{~V}$, the relation between $H$ and $W$ can be explicitly calculated (the blue line in Fig. 7). In Fig. 7, the theoretical relation between $H$ and $W$ is also compared to experimental results. The red data points show statistics of the pulse height from an exemplary measurement with water droplets of different sizes. The red dots give the average pulse height for pulses having the same pulse width (measured with the $1 \mu$ s resolution); the error bars give the standard deviation. It has to be mentioned that the measurement shown in Fig. 7 was obtained with the water droplets continuously growing with time as LACIS was cooled from ambient temperature down to the temperature of $-40^{\circ} \mathrm{C}$. This is usually done to induce ice formation on the tube wall before the experimental runs (as described in Niedermeier et al., 2010) and offers the possibility to investigate a wide range of droplet sizes. As a consequence, the thermodynamic conditions are not totally stable, resulting in slow perturbations of the flow profile inside the channel. Nevertheless, the figure shows the principle relationship between pulse width and pulse height that can be found in theory (see Eq. 8) and experiment.

Due to the nonlinear shape of the relationship between the pulse height and the pulse width (see Fig. 7), the form of the PWD is different from that of the PHD. For signal pulses smaller than $1 \mathrm{~V}$, a small change in the pulse amplitude results in a relatively large change in the pulse width. In contrast, for larger particles (signal $>1 \mathrm{~V}$ ), and, therefore, for larger pulse amplitudes, a relatively large difference in pulse amplitude results in a small variation of the pulse width. For bimodal distribution of pulse amplitudes (encountered in a typical ice nucleation experiment, later shown in Fig. 8), this leads to a broadening of the droplet mode and a more compact ice mode in $\mathrm{PWD}^{\mathrm{C}}$. For even larger droplets and ice particles, it is possible to reduce the amplification of the PMTs so that the signal resides within a suitable range of about $1 \mathrm{~V}$. 


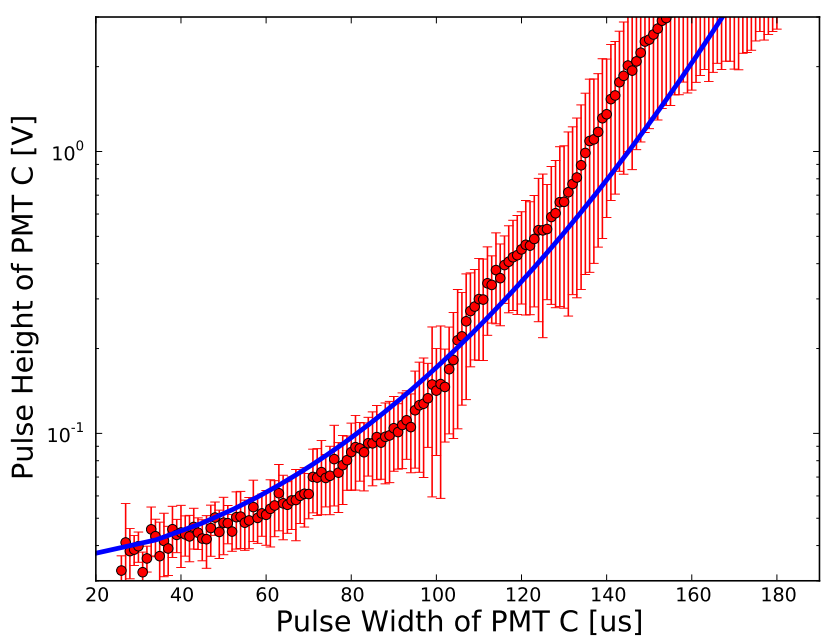

Fig. 7. Pulse width vs. pulse height for $\sigma_{\mathrm{p}}=28 \mu$ s and $L=0.03 \mathrm{~V}$ (blue line) and data from a measurement with water droplets of different sizes (red dots give the average pulse height for the pulses having the same width; error bars give the standard deviation).

To illustrate typical measurements with TOPS-Ice for droplets and ice, three examples are given in Fig. 8. Panels (a) and (b) show the PHD measured with PMT C $\left(\mathrm{PHD}^{\mathrm{C}}\right)$ and the $\mathrm{PWD}^{\mathrm{C}}$ for the droplet ensembles, respectively, obtained from an experiment where $200 \mathrm{~nm}$ ammonium sulfate particles activated to droplets of an approximate size of $2 \mu \mathrm{m}$. In this example, one narrow droplet mode exists both in $\mathrm{PHD}^{\mathrm{C}}$ and $\mathrm{PWD}^{\mathrm{C}}$. However, the $\mathrm{PHD}^{\mathrm{C}}$ in panel (a) is poorly resolved, whereas PWD $^{\mathrm{C}}$ in panel (b) shows a well-resolved main mode corresponding to the droplets and a shoulder on the left side of the droplet peak resulting from the droplets crossing the sensitive volume inside the edge zone that are not filtered out by the validation conditions. This resulting edge zone error is later considered as a measurement uncertainty (see Sect. 4.2).

Panels (c) and (d) show the $\mathrm{PHD}^{\mathrm{C}}$ and the $\mathrm{PWD}^{\mathrm{C}}$ for a particle ensemble consisting of only ice particles. In this particular experiment, the liquid droplets activated on the mineral dust particles (ATD, ISO 12103-1, A1 Ultrafine Test Dust, Powder Technology Inc., Burnsville, Minnesota, USA) froze at the temperature of $-40^{\circ} \mathrm{C}$, due to either heterogeneous or homogeneous ice nucleation. Both the $\mathrm{PHD}^{\mathrm{C}}$ and $\mathrm{PWD}^{\mathrm{C}}$ are very broad and show a clear single mode.

Panels (e) and (f) show, respectively, the $\mathrm{PHD}^{\mathrm{C}}$ and the $\mathrm{PWD}^{\mathrm{C}}$ for a mixed ensemble of droplets and ice particles formed on $300 \mathrm{~nm}$ ATD particles at $-35^{\circ} \mathrm{C}$. The characteristic size of ice particles in this example is similar to the size of the particles in the "ice only" experiment (panels c, d), and the size of the droplets in the mixture is similar to that of the "droplet only" experiment (panels a, b). Therefore, both the ice mode and the droplet mode are located in a similar range of magnitudes compared to the "ice only" and "droplet only" experiments. As a result, the PWD ${ }^{\mathrm{C}}$ shows two clearly distinguishable modes, whereas the $\mathrm{PHD}^{\mathrm{C}}$ exhibits a narrow droplet mode and inhomogeneously scattered discrete values corresponding to ice particles. This mode separation suggests an advantage of using the $\mathrm{PWD}^{\mathrm{C}}$ for the retrieval of ice fractions in the mixed-phase experiments. Finally, the number of droplets and ice particles can be obtained and the ice fraction $f_{\text {ice }}$ can be reliably calculated by an algorithm explained in Sect. 4.2.

\subsection{Evaluation of the TOPS-Ice data}

In the following, the calculation of the ice fraction from the PWD $^{\mathrm{C}}$ measured with TOPS-Ice is described in detail. The applicability of the method is demonstrated in two immersion freezing experiments with different resulting ice fractions performed at LACIS

First, a digital low-pass filter with Hamming window is applied to the $\mathrm{PWD}^{\mathrm{C}}$. The resulting smoothed $\mathrm{PWD}^{\mathrm{C}}$ is shown by the black line in Fig. 9. Next, the following iteration procedure is applied to the data: at the initial step, a normal distribution with parameters $N_{0}, \sigma_{0}$ and $\mu_{0}$ is fitted to the droplet mode of the smoothed PWD ${ }^{\mathrm{C}}$, where $N_{0}$ is the area under the curve, $\sigma_{0}$ is the standard deviation and $\mu_{0}$ is the mean value of the normal distribution (similar to Eq. 5). To ensure that the droplet mode, and not the ice mode, is used for the fit, at the initial step the data range is manually constrained to the droplet mode. For every next iteration step $n$, only a limited range $W_{n}$ of the $\mathrm{PWD}^{\mathrm{C}}$ data is considered:

$\mu_{n-1}-k \sigma_{n-1}<W_{n}<\mu_{n-1}+l \sigma_{n-1}$,

where $\mu_{n-1}$ and $\sigma_{n-1}$ are taken from the previous iteration fit and the two parameters $k$ and $l$ are set by default to 1.5 , which mostly lead to a good agreement of the fitting curves to the filtered data. After limitation of the data to $W_{n}$, a new fit with parameters $N_{n}, \sigma_{n}$ and $\mu_{n}$ is applied.

If $\sigma_{n}-\sigma_{n-1} \leq 0.0001$, the iteration is stopped, the number of droplets $N_{\mathrm{d}}$ is set to $N_{n}$, and $\sigma_{\mathrm{d}}=\sigma_{n}$ and $\mu_{\mathrm{d}}=\mu_{n}$ are the parameters of the droplet mode (red line in Fig. 9). This iteration algorithm ensures that the normal distribution fit is applied to the droplet mode only.

Next, the fitted droplet mode is subtracted from the smoothed $\mathrm{PWD}^{\mathrm{C}}$, thus leaving the residual data shown by the blue line in Fig. 9. The residual data shows two major modes. The mode on the right side is assumed to be produced by the ice particles, and is used for the calculation of the ice fraction $f_{\text {ice. }}$. The mode on the left side is caused by the edge zone counts coming from ice and droplets; the number of these counts $N_{\mathrm{f}}$ allows us to estimate the error of the ice fraction $f_{\text {ice }}$.

The number of ice particles $N_{\text {ice }}$ is determined by summing up over the ice mode of the residual data. The ice fraction $f_{\text {ice }}$ is then given by

$f_{\text {ice }}=\frac{N_{\text {ice }}}{N_{\mathrm{d}}+N_{\text {ice }}}$. 

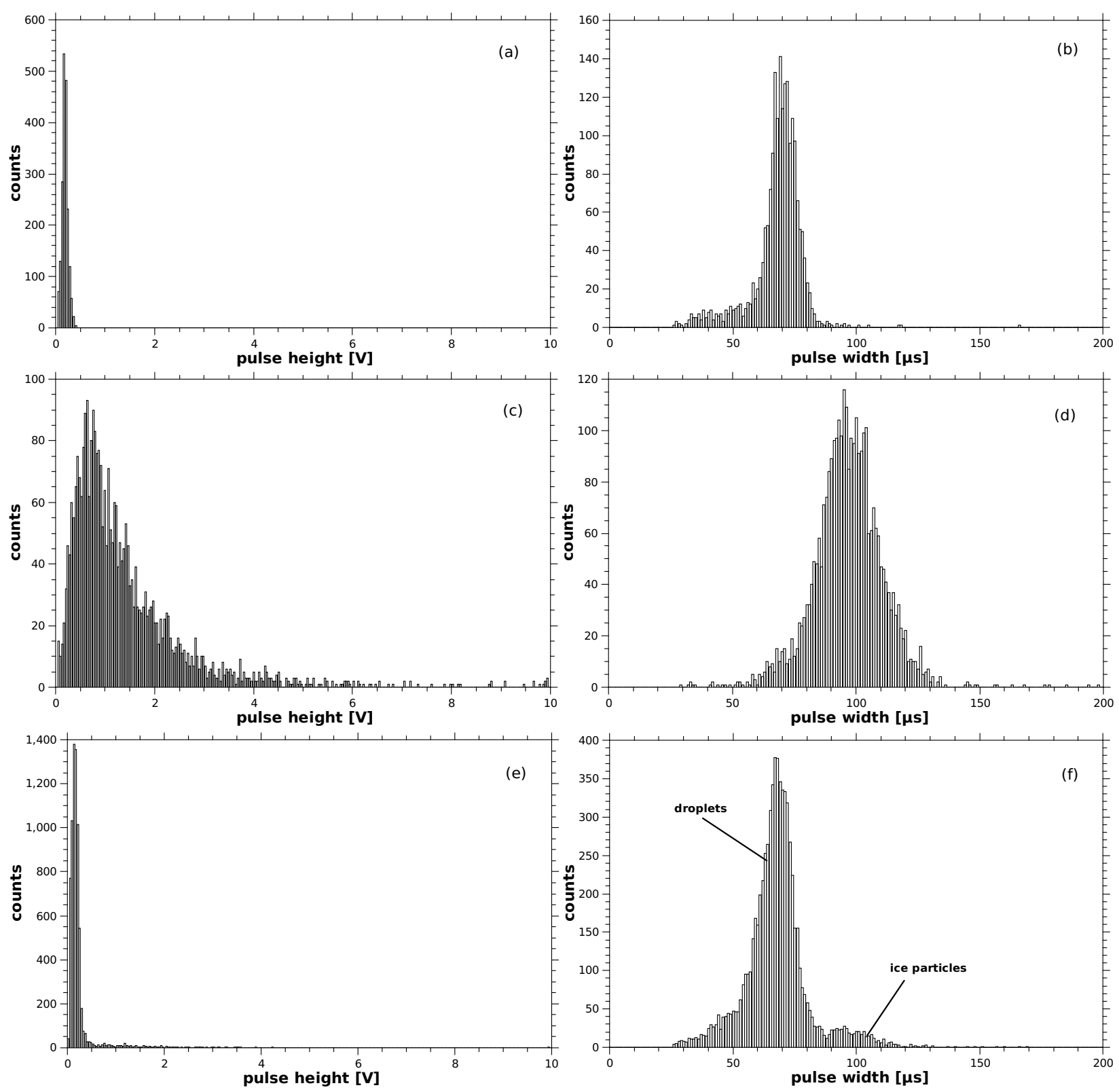

Fig. 8. Pulse height (left column) and pulse width (right column) distributions recorded by PMT C for different particle populations. Panels $(a, b)$ show the distributions measured for the population of monodisperse droplets of approximately $2 \mu$ m in diameter. Panels (c, d) show the distributions recorded for ice particles formed as a result of droplets freezing at $-40^{\circ} \mathrm{C}$. Panels (e, f) show the distributions produced by a mixed particle ensemble of liquid and frozen droplets formed on $300 \mathrm{~nm}$ Arizona Test Dust (ATD) particles at $-35^{\circ} \mathrm{C}$.

The absolute error from the edge zone error counts is $N_{\mathrm{f}}$. Having no information about what fraction of edge zone error counts comes from what particle mode, we take $N_{\mathrm{f}}$ as an upper estimate of the absolute error for the number of unfrozen droplets as $\Delta N_{\mathrm{d}}=N_{\mathrm{f}} N_{\mathrm{d}} /\left(N_{\mathrm{d}}+N_{\text {ice }}\right)$ and as an upper estimate of the absolute error for the number of ice particles as $\Delta N_{\text {ice }}=N_{\mathrm{f}} N_{\text {ice }} /\left(N_{\mathrm{d}}+N_{\text {ice }}\right)$. This approach gives us an upper estimate of the relative error of $f_{\text {ice }}$ in the following form:
$\frac{\Delta f_{\text {ice }}}{f_{\text {ice }}}=\frac{2 N_{\mathrm{f}} N_{\mathrm{d}}}{\left(N_{\mathrm{d}}+N_{\text {ice }}\right)^{2}}$

This method was applied to extract the $f_{\text {ice }}$ values from the measurements done with TOPS-Ice installed underneath LACIS, for immersion freezing studies with $300 \mathrm{~nm}$ ATD particles. The values of $f_{\text {ice }}$ obtained with the two different optical instruments (WELAS, as described in Niedermeier et al., 2010, and TOPS-Ice, this manuscript) are compared in Fig. 10. 

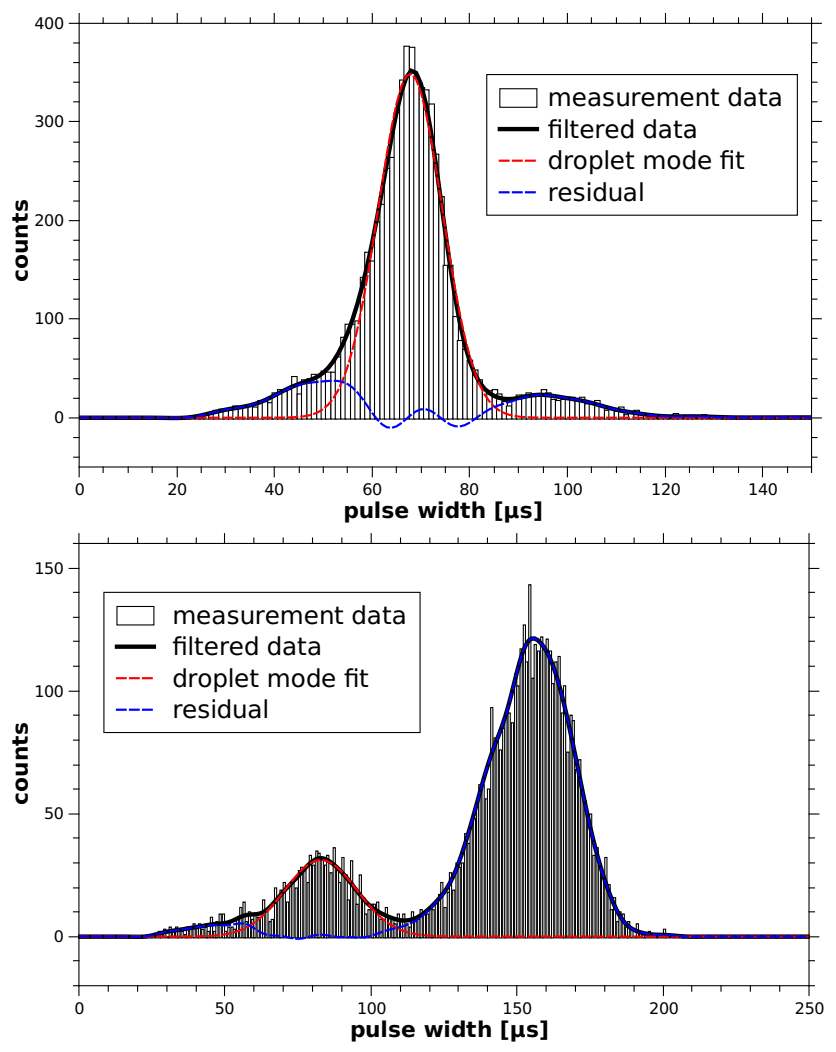

Fig. 9. Illustration of the data evaluation algorithm for two different cases, $f_{\text {ice }}=0.06$ in top panel (example from Fig. 4.1 ), $f_{\text {ice }}=0.82$ in bottom panel.

During the measurements where TOPS-Ice was used for the determination of $f_{\text {ice, }}$ LACIS was run in the mode where droplets and ice particles coexist at the outlet of the LACIS flow tube. The resulting ice fraction is shown as red diamonds. In contrast, for the measurements with WELAS (blue squares in Fig. 10), LACIS was run in a mode where the liquid droplets have been evaporated before reaching the end of the flow tube. The two modes of operation differ in a different dew point, adjusted at the inlet of the tube, which results in either droplets and ice particles (measurement with TOPSIce) or dry dust particles and ice particles (measurement with WELAS) at the outlet of LACIS. The error bars of the TOPSIce data show the experimental uncertainties resulting from the edge zone counts, as explained above. The error bars of the WELAS data are the doubled standard deviation taken from different measurements. Although LACIS was run in two different modes of operation and two completely different optical methods for the detection were used, the figure shows an excellent agreement of both measurement results. This strongly underlines the feasibility of TOPS-Ice to distinguish between droplets and ice particles and hence its ability to determine ice fractions in LACIS.

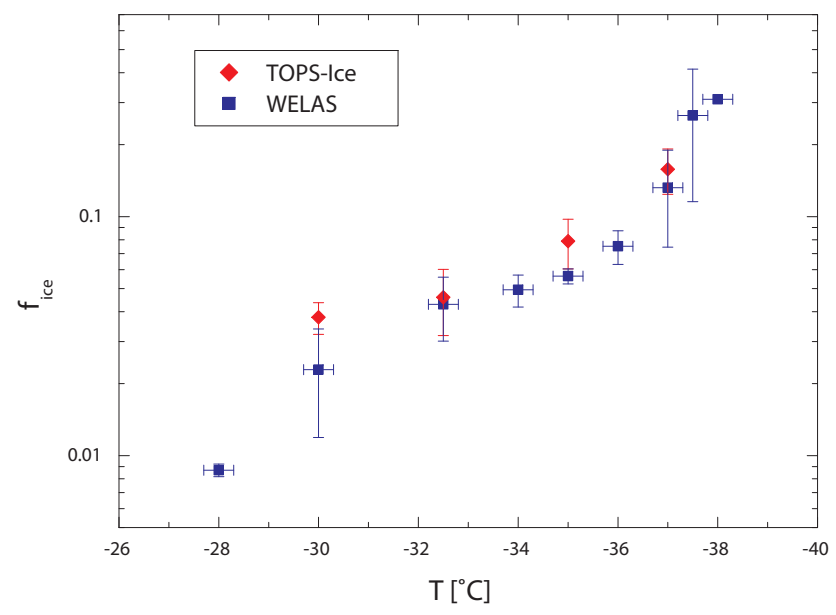

Fig. 10. Comparison of the ice fraction vs. freezing temperature determined with WELAS (Niedermeier et al., 2010, 2011) and with TOPS-Ice. $300 \mathrm{~nm}$ Arizona Test Dust particles were used for both measurements.

\section{Conclusions}

An optical single-particle counter TOPS-Ice was developed to satisfy the need for reliable determination of the ice particle fraction in mixed particle ensembles consisting of liquid droplets and ice particles of similar size. The applicability of the method has been demonstrated in the experiment conducted at the laminar flow diffusion chamber LACIS.

The differentiation between liquid and frozen droplets is based on the measurement of the cross-polarized component of the scattered light. Although for the chosen scattering geometry $\left((42.5 \pm 12.7)^{\circ}\right.$ from the forward direction) the crosspolarized linear component of the light scattered by spherical droplets is not completely suppressed, the non-sphericity of ice particles results in a stronger scattering signal than for the droplets, which can be employed for the differentiation of ice and droplet modes.

To support these experimental findings, we have used the T-matrix method to calculate the scattering intensities for the ensembles of spheroids of variable aspect ratio in random orientation with account for the actual scattering geometry of TOPS-Ice. We show that the scattering intensity of a spherical particle in the depolarization channel is weaker than for the non-spherical particles and this difference can be used for differentiation. However, this is only valid for the statistically large mixed ensembles of spherical droplets and randomly oriented ice particles.

The usage of the scattering signal pulse width $W$ of the depolarization channel as a measurement parameter instead of the signal pulse amplitude turned out to be advantageous in our measurement regime. Exploiting the nonlinear relationship between pulse width and pulse amplitude, we show that for a mixed particle ensemble the distribution of the signal pulse width measured in the depolarization channel always 
has two modes: one for the liquid water droplets and one for the ice particles. Assuming that the ice particle mode always corresponds to the pulses with higher amplitudes and therefore greater width, and applying a Gaussian fit routine to the two modes, both the number of liquid droplets and ice particles, and consequently $f_{\text {ice }}$, can be determined.

However, one can think of some improvements concerning further types of TOPS-Ice; e.g., calculations show that the choice of a different scattering angle at $100^{\circ}$ may raise the signal of the ice particles and increase the difference between the droplet and the ice particle distributions.

To verify the applicability of TOPS-Ice, we measured the fractions of frozen droplets as a function of temperature in the immersion freezing experiment in LACIS. The values obtained with TOPS-Ice and the data evaluation algorithm developed were compared with results obtained from a different optical instrument, the white light optical particle counter WELAS, as described in Niedermeier et al. (2010). Both methods show an excellent agreement although LACIS was run in two different modes of operation and the optical detection methods were completely different.

The measuring principle of the newly developed TOPS-Ice instrument has certain advantages as compared to the WELAS instrument. In the WELAS-based measurements, the droplets have to be evaporated within LACIS, so that only small aerosol particles and large ice particles reach the optical detection section at the outlet of the flow tube. In the case of large or strongly polydisperse aerosol particles, the dry aerosol mode overlaps with the ice particle mode, making the mode separation not possible. In such a situation, allowing the coexistence of droplets and ice particles at the LACIS outlet and applying the depolarization-based detection method is the only way to separate the modes and to enable the $f_{\text {ice }}$ determination.

Acknowledgements. This work was partly funded by the German Science Foundation (DFG) under contract HE 939/21-1. Additionally, this work was financially supported by the research project EUROCHAMP II.

Edited by: S. Malinowski

\section{References}

Ansmann, A., Tesche, M., Althausen, D., Müller, D., Seifert, P., Freudenthaler, V., Heese, B., Wiegner, M., Pisani, G., Knippertz, P., and Dubovik, O.: Influence of Saharan dust on cloud glaciation in southern Morocco during the Saharan Mineral Dust Experiment, J. Geophys. Res.-Atmos., 113, D04210, doi:10.1111/j.1600-0889.2008.00384.x, 2008.

Ansmann, A., Tesche, M., Seifert, P., Althausen, D., Engelmann, R., Fruntke, J., Wandinger, U., Mattis, I., and Müller, D.: Evolution of the ice phase in tropical altocumulus: SAMUM lidar observations over Cape Verde, J. Geophys. Res.-Atmos., 114, D17208, doi:10.1029/2008JD011659, 2009.
Bailey, M. and Hallett, J.: A comprehensive habit diagram for atmospheric ice crystals: Confirmation from the laboratory, AIRS II, and other field studies, J. Atmos. Sci., 66, 2888-2899, 2009.

Baran, A. J., Yang, P., and Havemann, S.: Calculation of the single-scattering properties of randomly oriented hexagonal ice columns: a comparison of the T-matrix and the finite-difference time-domain methods, Appl. Optics, 40, 4376-4386, 2001.

Bohren, C. F. and Huffmann, D. R.: Absorption and Scattering of Light by Small Particles, Wiley, 1983.

Bundke, U., Nillius, B., Jaenicke, R., Wetter, T., Klein, H., and Bingemer, H.: The fast Ice Nucleus chamber FINCH, Atmos. Res., 90, 180-186, Sp. Iss. SI, 2008.

Cantrell, W. and Heymsfield, A.: Production of ice in tropospheric clouds - A review, B. Am. Meteorol. Soc., 86, 795-807, 2005.

de Boer, G., Morrison, H., Shupe, M., and Hildner, R.: Evidence of liquid dependent ice nucleation in high-latitude stratiform clouds from surface remote sensors, Geophys. Res. Lett., 38, L01803, doi:10.1029/2010GL046016, 2011.

Draine, B. and Flatau, P.: Discrete-dipole approximation for scattering calculations, J. Opt. Soc. Am. A, 11, 1491-1499, 1994.

Glen, A. and Brooks, S. D.: A new method for measuring optical scattering properties of atmospherically relevant dusts using the Cloud and Aerosol Spectrometer Polarization (CASPOL), Atmos. Chem. Phys., 13, 1345-1356, doi:10.5194/acp-13-13452013, 2013.

Hartmann, S., Niedermeier, D., Voigtländer, J., Clauss, T., Shaw, R. A., Wex, H., Kiselev, A., and Stratmann, F.: Homogeneous and heterogeneous ice nucleation at LACIS: operating principle and theoretical studies, Atmos. Chem. Phys., 11, 1753-1767, doi:10.5194/acp-11-1753-2011, 2011.

Kiselev, A., Wex, H., Stratmann, F., Nadeev, A., and Karpushenko, D.: White-light optical particle spectrometer for in situ measurements of condensational growth of aerosol particles, Appl. Optics, 44, 4693-4701, 2005.

Krämer, M., Meyer, J., Afchine, A., Newton, R., Baumgardner, D., and Schnaiter, M.: HALO ice crystal spectrometer intercomparison at the AIDA - chamber: first results from the novel ice experiment NIXE-CAPS, Geophys. Res. Abstr., EGU2009-3720-1, EGU General Assembly 2009, Vienna, Austria, 2009.

Macke, A., Mueller, J., and Raschke, E.: Single scattering properties of atmospheric ice crystals, J. Atmos. Sci., 53, 2813-2825, 1996.

Mishchenko, M. I. and Hovenier, J. W.: Depolarization of Light Backscattered by Randomly Oriented Nonspherical Particles, Opt. Lett., 20, 1356-1358, 1995.

Mishchenko, M. I. and Sassen, K.: Depolarization of lidar returns by small ice crystals: An application to contrails, Geophys. Res. Lett., 25, 309-312, 1998.

Nicolet, M., Stetzer, O., Lüönd, F., Möhler, O., and Lohmann, U.: Single ice crystal measurements during nucleation experiments with the depolarization detector IODE, Atmos. Chem. Phys., 10, 313-325, doi:10.5194/acp-10-313-2010, 2010.

Nicolet, M., Schnaiter, M., and Stetzer, O.: Circular depolarization ratios of single water droplets and finite ice circular cylinders: a modeling study, Atmos. Chem. Phys., 12, 4207-4214, doi:10.5194/acp-12-4207-2012, 2012.

Niedermeier, D., Hartmann, S., Shaw, R. A., Covert, D., Mentel, T. F., Schneider, J., Poulain, L., Reitz, P., Spindler, C., Clauss, T., Kiselev, A., Hallbauer, E., Wex, H., Mildenberger, K., and Stratmann, F.: Heterogeneous freezing of droplets with immersed 
mineral dust particles - measurements and parameterization, Atmos. Chem. Phys., 10, 3601-3614, doi:10.5194/acp-10-36012010, 2010.

Niedermeier, D., Hartmann, S., Clauss, T., Wex, H., Kiselev, A., Sullivan, R. C., DeMott, P. J., Petters, M. D., Reitz, P., Schneider, J., Mikhailov, E., Sierau, B., Stetzer, O., Reimann, B., Bundke, U., Shaw, R. A., Buchholz, A., Mentel, T. F., and Stratmann, F.: Experimental study of the role of physicochemical surface processing on the IN ability of mineral dust particles, Atmos. Chem. Phys., 11, 11131-11144, doi:10.5194/acp-11-11131-2011, 2011.

Prenni, A. J., DeMott, P. J., Rogers, D. C., Kreidenweis, S. M., McFarquhar, G. M., Zhang, G., and Poellot, M. R.: Ice nuclei characteristics from M-PACE and their relation to ice formation in clouds, Tellus B, 61, 436-448, doi:10.1111/j.16000889.2009.00415.x, 2009.

Pruppacher, H. R. and Klett, J. D.: Microphysics of Clouds and Precipitation, Kluwer Academic Publishers, Dordrecht, The Netherlands, 1997.

Rogers, D.: Development of a continuous flow thermal gradient diffusion chamber for ice nucleation studies, Atmos. Res., 22, 149$181,1988$.

Rother, T.: Electromagnetic Wave Scattering on Nonspherical Particles: Basic Methodology and Simulations, Springer Berlin Heidelberg, 2009.

Sassen, K.: The Polarization Lidar Technique for Cloud Research - a Review and Current Assessment, B. Am. Meteorol. Soc., 72, 1848-1866, 1991.

Schmidt, S., Lehmann, K., and Wendisch, M.: Minimizing instrumental broadening of the drop size distribution with the M-FastFSSP, J. Atmos. Ocean. Tech., 21, 1855-1867, 2004.

Schnaiter, M., Büttner, S., Skrotzki, J., Vragel, M., and Wagner, R.: Influence of particle size and shape on the backscattering linear depolarisation ratio of small ice crystals - cloud chamber measurements in the context of contrail and cirrus microphysics, Atmos. Chem. Phys., 12, 10465-10484, doi:10.5194/acp-1210465-2012, 2012

Seifert, P., Ansmann, A., Mattis, I., Wandinger, U., Tesche, M., Engelmann, R., Müller, D., Pérez, C., and Haustein, K.: Saharan dust and heterogeneous ice formation: Eleven years of cloud observations at a central European EARLINET site, J. Geophys. Res.-Atmos., 115, D20201, doi:10.1029/2009JD013222, 2010.
Stetzer, O., Baschek, B., Lüönd, F., and Lohmann, U.: The Zurich Ice Nucleation Chamber (ZINC) - A new instrument to investigate atmospheric ice formation, Aerosol Sci. Technol., 42, 6474, 2008.

Stratmann, F., Kiselev, A., Wurzler, S., Wendisch, M., Heintzenberg, J., Charlson, R. J., Diehl, K., Wex, H., and Schmidt, S.: Laboratory studies and numerical simulations of cloud droplet formation under realistic supersaturation conditions, J. Atmos. Ocean. Tech., 21, 876-887, 2004.

Takano, Y. and Jayaweera, K.: Scattering Phase Matrix for Hexagonal Ice Crystals Computed from Ray Optics, Appl. Optics, 24, 3254-3263, 1985.

van de Hulst, H. C.: Light Scattering by Small Particles, Dover Pubn Inc, 1982.

Wagner, R., Linke, C., Naumann, K. H., Schnaiter, M., Vragel, M., Gangl, M., and Horvath, H.: A review of optical measurements at the aerosol and cloud chamber AIDA, J. Quant. Spectrosc. Ra., 110, 930-949, 2009.

Wiacek, A., Peter, T., and Lohmann, U.: The potential influence of Asian and African mineral dust on ice, mixed-phase and liquid water clouds, Atmos. Chem. Phys., 10, 8649-8667, doi:10.5194/acp-10-8649-2010, 2010.

Wriedt, T. and Hellmers, J.: New Scattering Information Portal for the light-scattering community, J. Quant. Spectrosc. Ra., 109, 1536-1542, 2008.

Yang, P. and Liou, K.: Finite difference time domain method for light scattering by small ice crystals in three-dimensional shape, J. Opt. Soc. Am. A, 13, 2072-2085, 1996.

Yang, P., Wei, H. L., Kattawar, G. W., Hu, Y. X., Winker, D. M., Hostetler, C. A., and Baum, B. A.: Sensitivity of the backscattering Mueller matrix to particle shape and thermodynamic phase, Appl. Optics, 42, 4389-4395, 2003.

Zakharova, N. T. and Mishchenko, M. I.: Scattering properties of needlelike and platelike ice spheroids with moderate size parameters, Appl. Optics, 39, 5052-5057, 2000. 